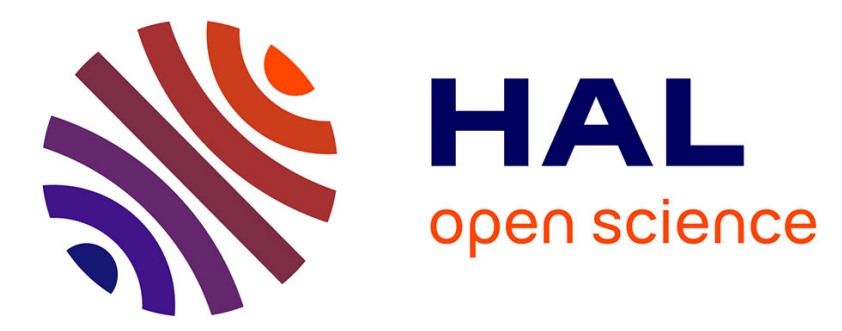

\title{
Physics-based Deep Neural Network for Real-Time Lesion Tracking in Ultrasound-guided Breast Biopsy
}

\author{
Andrea Mendizabal, Eleonora Tagliabue, Jean-Nicolas Brunet, Diego
}

Dall'Alba, Paolo Fiorini, Stéphane Cotin

\section{- To cite this version:}

Andrea Mendizabal, Eleonora Tagliabue, Jean-Nicolas Brunet, Diego Dall'Alba, Paolo Fiorini, et al.. Physics-based Deep Neural Network for Real-Time Lesion Tracking in Ultrasound-guided Breast Biopsy. Computational Biomechanics for Medicine XIV, Oct 2019, Shenzhen, China. hal-02311277

\section{HAL Id: hal-02311277 \\ https://inria.hal.science/hal-02311277}

Submitted on 29 Oct 2019

HAL is a multi-disciplinary open access archive for the deposit and dissemination of scientific research documents, whether they are published or not. The documents may come from teaching and research institutions in France or abroad, or from public or private research centers.
L'archive ouverte pluridisciplinaire HAL, est destinée au dépôt et à la diffusion de documents scientifiques de niveau recherche, publiés ou non, émanant des établissements d'enseignement et de recherche français ou étrangers, des laboratoires publics ou privés. 


\title{
Physics-based Deep Neural Network for Real-Time Lesion Tracking in Ultrasound-guided Breast Biopsy
}

\author{
Andrea Mendizabal ${ }^{1, *}$, Eleonora Tagliabue ${ }^{2, *}$, Jean-Nicolas Brunet ${ }^{1}$, Diego \\ Dall'Alba $^{2}$, Paolo Fiorini' ${ }^{2}$, and Stéphane Cotin ${ }^{1}$ \\ 1 INRIA Strasbourg \\ andrea.mendizabal@inria.fr \\ 2 Università degli Studi di Verona \\ eleonora.tagliabue@univr.it \\ * A. Mendizabal and E. Tagliabue contributed equally to the paper.
}

\begin{abstract}
In the context of ultrasound (US) guided breast biopsy, image fusion techniques can be employed to track the position of USinvisible lesions previously identified on a pre-operative image. Such methods have to account for the large anatomical deformations resulting from probe pressure during US scanning within the real-time constraint. Although biomechanical models based on the finite element (FE) method represent the preferred approach to model breast behavior, they cannot achieve real-time performances. In this paper we propose to use deep neural networks to learn large deformations occurring in ultrasoundguided breast biopsy and then to provide accurate prediction of lesion displacement in real-time. We train a U-Net architecture on a relatively small amount of synthetic data generated in an offline phase from FE simulations of probe-induced deformations on the breast anatomy of interest. Overall, both training data generation and network training are performed in less than 5 hours, which is clinically acceptable considering that the biopsy can be performed at most the day after the pre-operative scan. The method is tested both on synthetic and on real data acquired on a realistic breast phantom. Results show that our method correctly learns the deformable behavior modelled via $\mathrm{FE}$ simulations and is able to generalize to real data, achieving a target registration error comparable to that of FE models, while being about a hundred times faster.
\end{abstract}

Keywords: Ultrasound-guided Breast Biopsy · Deep Neural Networks - Real-time Simulation.

\section{Introduction}

Breast biopsy is the preferred technique to evaluate the malignancy of screeningdetected suspicious lesions. To direct the needle towards the target, biopsy procedures are performed under image guidance, normally done with ultrasound (US) probes due to their ability to provide real-time visualization of both the 
needle and the internal structures [18]. However, proper needle placement with US remains a challenging task. First, malignant lesions cannot always be adequately visualized due to the poor image contrast of US. Furthermore, navigation towards complex 3D lesion geometries is commonly achieved using 2D freehand US (FUS) systems, which provide information in a lower-dimensional space [11]. Since highly sensitive pre-operative images (such as MRI or CT) can provide accurate positions of the lesions, finding a method to update these positions from real-time US images during an intervention would highly benefit current biopsy procedures. Several commercial and research platforms have implemented image fusion techniques that align pre-operative and intra-operative data, exploiting rigid or affine registration methods [6]. However, when dealing with breast anatomy, large deformations arise due to compression forces applied by the US probe. To provide accurate probe-tissue coupling and acceptable image quality, an appropriate alignment procedure of the pre-operative and US data is required.

Accurate modelling of soft tissue deformation in real-time is a far-from-beingsolved problem. Biomechanical models relying on the finite element method (FEM) realistically calculate soft tissue deformations by using a mathematical model based on continuum mechanics theory. Although these models have been successfully employed for multimodal breast image registration, they have never been applied to registration between pre-operative data and intra-operative US, due to difficulties in providing a prediction within real-time constraints [8]. This is especially true when considering large, non-linear deformations which involve hyperelastic objects, as it is the case for the breast.

In order to meet real-time compliance, various techniques have been proposed to simplify the computational complexity of FEM. Some of them have focused on optimizing linear solvers (the main bottleneck of FEM) or the formulation itself, such as corotational [5] and multiplicative jacobian energy decomposition [13]. Very efficient implementations also exist, like Total Lagrangian explicit dynamics (TLED) [15], which can achieve real-time performances when coupled with explicit time integration and GPU-based solvers [10]. Another possible option to lower the simulation time is through dimensionality reduction techniques, like Proper Orthogonal Decomposition (POD), where the solution to a highdimensional problem is encoded as a subset of precomputed modes. The most optimized approach used to model breast biomechanics is the one proposed by Han et al. in [7], which relies on GPU-based TLED formulation. Despite the significant simulation speedup achieved, solving the FE system took around 30s, which is still not compatible with real-time. Modelling methods that do not rely on continuum mechanics laws have also been used to approximate soft tissues behavior. Among these, the position-based dynamics (PBD) approach has been used to predict breast lesions displacement due to US probe pressure in realtime, providing comparable accuracy with FE models [21]. However, not being based on real mechanical properties, such model requires an initial optimization of simulation parameters to obtain a realistic description of the deformation. 
An emerging approach which has the potential of being both accurate and fast, exploits neural networks to estimate soft tissue behavior. Machine learningbased methods have proven successful to predict the entire 3D organ deformation starting either by applied surface forces $[17,22]$ or by acquired surface displacements $[19,1]$. Being networks trained with synthetic data generated from FE simulations, they can reproduce a realistic physics-based description of the organ mechanical behavior. Using FE simulations for model training in the context of MRI-US deformable image registration has already been proposed in [9], where the authors build a statistical model of prostate motion which can account for different properties and boundary conditions. In the case of the breast, the potentiality of employing machine learning techniques has been already shown in [14], where several tree-based methods have been employed to estimate breast deformation due to compression between biopsy plates. These methods have been trained on 10 different patient geometries with a very specific FE simulation, where the upper plate is displaced vertically towards the lower one.

Similarly to works in $[19,1]$, we propose an approach where a neural network is trained to predict the deformation of internal breast tissues starting from the acquired surface displacements induced by the US probe. Our network can be seen as a patient-specific model. We train it on a single patient geometry before surgery, with a relatively small amount of training data. However, in contrast to the work of [14], FE simulations that compose the training set are generated with several random input displacements, making our approach able to generalize to different probe positions and compression extents.

The proposed method consists in a U-Net architecture, described in Sect. 2.2, and an immersed boundary method used for generating patient-specific simulations, described in Sect. 2.3. Results presented in Sect. 3 show the efficiency of the method when applied to both synthetic and ex vivo scenarios. Our contribution consists of a novel method to generate a real-time capable soft tissue model to improve target visualization during needle-based procedures. The position of lesions identified beforehand on pre-operative images can be updated from intra-operative ultrasound data and visualized by the surgeon in real-time.

\section{Methods}

This work presents a data-driven method to estimate in real-time the displacement of the breast internal structures due to probe pressure during US scanning. In our pipeline, we assume to have a patient-specific geometric model of the breast, obtained from pre-operative imaging such as MRI, and to know the position and orientation of the US probe at each time, thanks to a spatial tracking system. If the tracking coordinate system and the coordinate system of pre-operative imaging are registered, knowledge about the $3 \mathrm{D}$ pose and the geometry of the US probe directly allows to identify the contact surface between the breast and the probe. Since the US probe is represented as a rigid body, we can reasonably assume that when the anatomy is deformed by the probe during the image acquisition process, points on the breast surface below the US 
probe will be displaced to the same exact extent as the probe itself. As a consequence, our method can predict the displacements of all the points within the anatomy given as input the displacement of the surface nodes in contact with the US probe. The decision of relying on surface displacement inferred from the spatial tracking of the US probe instead of directly tracking surface deformations (through, for example, an RGBD camera) was taken from the fact that probe-induced deformations are large but local, and the probe itself would occlude most of the deformed surface to the sensor, thus preventing an accurate estimation of the contact surface displacements.

\subsection{The U-Net architecture}

The objective of our work is to find the relation function $f$ between the partial surface deformation under the US probe and the deformation inside the breast. Let $\mathbf{u}_{\mathbf{s}}$ be the surface deformation and $\mathbf{u}_{\mathbf{v}}$ the volumetric displacement field. In order to find $f$ a minimization is performed on the expected error over a training set $\left\{\left(\mathbf{u}_{\mathbf{s}}{ }^{n}, \mathbf{u}_{\mathbf{v}}{ }^{n}\right)\right\}_{n=1}^{N}$ of $N$ samples:

$$
\min _{\theta} \frac{1}{N} \sum_{n=1}^{N}\left\|f\left(\mathbf{u}_{\mathbf{s}}{ }^{n}\right)-\mathbf{u}_{\mathbf{v}}{ }^{n}\right\|_{2}^{2}
$$

where $\theta$ is the set of parameters of the network $f$. We propose to use the same architecture as in [1], that is a U-Net [20] adapted to our application (see Fig. 1). The network consists of an encoding path that reduces the high dimensional input into a reduced space, and a decoding path that expands it back to the original shape. The skip connections transfer features along matching levels from the encoding path to the decoding path through crop and copy operations. As

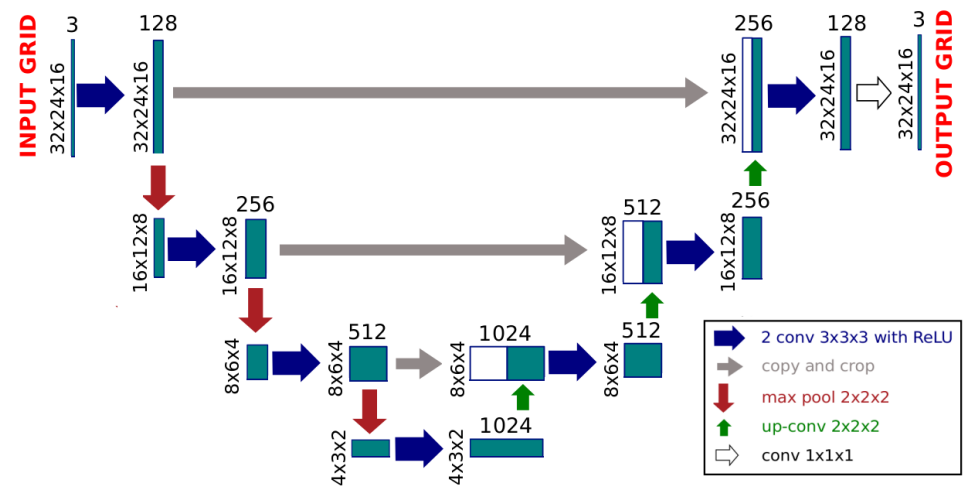

Fig. 1. U-Net architecture for a padded input grid of size $32 \times 24 \times 16$.

Fig. 1 shows, the encoding path consists of $k$ sequences ( $k=3$ in our case) 
of two padded $3 \times 3 \times 3$ convolutions and a $2 \times 2 \times 2$ max pooling operation. At each step, each feature map doubles the number of channels and halves the spatial dimensions. In the lower part of the U-Net there are two extra $3 \times 3 \times 3$ convolutional layers leading to a 1024-dimensional array. In a symmetric manner, the decoding path consists of $k$ sequences of an up-sampling $2 \times 2 \times 2$ transposed convolution followed by two padded $3 \times 3 \times 3$ convolutions. At each step of the decoding path, each feature map halves the number of channels and doubles the spatial dimensions. There is a final $1 \times 1 \times 1$ convolutional layer to transform the last feature map to the desired number of channels of the output ( 3 channels in our case). The design of the U-Net is based on a grid-like structure due to this up- and down-sampling process. Hence we directly mesh our deformable object with regular hexahedral elements as explained in the next section.

\subsection{Simulation of breast tissue using hexahedral grids}

The training data set consists of pairs of $\left(\mathbf{u}_{\mathbf{s}}, \mathbf{u}_{\mathbf{v}}\right)$ where $\mathbf{u}_{\mathbf{s}}$ is the input partial surface displacement and $\mathbf{u}_{\mathbf{v}}$ is the volumetric displacement field. Even though the data generation process takes place in an offline phase, in order to generate enough training data with FE simulations within clinically acceptable times (the intervention can be performed on the day after pre-operative scan is acquired), it is important to have simulations that are both accurate and computationally efficient.

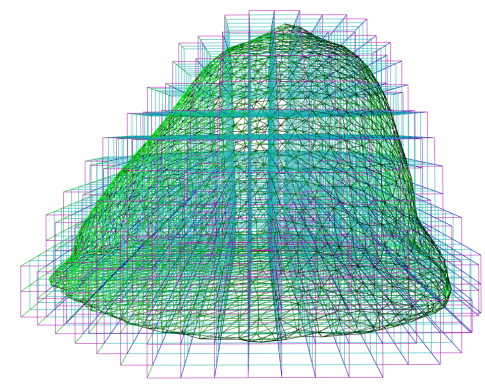

Fig. 2. Breast surface mesh obtained from a pre-operative CT scan immersed in a hexahedral grid for FEM computations.

We consider the boundary value problem of computing the deformation on a domain $\Omega$ under both Dirichlet and Neumann boundary conditions. Let $\Gamma$ be the boundary of $\Omega$ (in our case, $\Gamma$ corresponds to breast external surface, while $\Omega$ represents the entire breast volume). We assume that Dirichlet boundary conditions are applied to $\Gamma_{D}$ and are a-priori known, whereas Neumann boundary conditions are applied to $\Gamma_{N}$, a subset of $\Gamma$ that represents probe-tissue contact area and changes depending on current US probe position. In this work, training data for the network are generated by solving the discretized version of the 
following boundary value problem, exploiting the FE method:

$$
\left\{\begin{array}{l}
-\boldsymbol{\nabla} \cdot \boldsymbol{\sigma}=\mathbf{0} \text { in } \Omega \\
\mathbf{u}=0 \text { on } \Gamma_{D} \\
\boldsymbol{\sigma} \mathbf{n}=\mathbf{t} \text { on } \Gamma_{N}
\end{array}\right.
$$

where $\boldsymbol{\sigma}$ is the Cauchy stress tensor, $\mathbf{n}$ is the unit normal to $\Gamma_{N}$ and $\mathbf{t}$ is a traction force applied to the boundary. Note that in (2) we neglect all timedependent terms and we do not apply any body force like gravity, since our geometric model already accounts for the effect of gravity force. The relation between stress and strain is described through the Saint Venant-Kirchhoff model, which is the simplest and most efficient extension of a linear elastic material to the nonlinear regime. This choice is motivated by the fact that a simple linear elastic model would not be able to appropriately describe the large deformations undergone by the breast. An iterative Newton-Raphson method is used to solve the non-linear system of equations approximating the unknown displacement.

We choose to discretize the domain into 8-node hexahedral elements not only for their good convergence properties and lock-free behavior, but also because it is the required structure for the input to the network. To do that, the $3 \mathrm{D}$ breast geometry is embedded in a regular grid of hexahedral elements (see Fig. 2) and we use an immersed-boundary method to correctly approximate the volume of the object in the FE method computations.

\subsection{Data generation}

The input to the network corresponds to the displacement $\mathbf{u}_{\mathbf{s}}$ of the points belonging to the breast-probe contact area. The punctual displacements are spread to the nodes of the surrounding cuboid cell through a barycentric mapping and the corresponding volume displacement $\mathbf{u}_{\mathbf{v}}$ is obtained by the previously explained FE approach in response to $\mathbf{u}_{\mathbf{s}}$. The data used to train the network must be representative of the application scenario and must allow the network to extract the pertinent features of the tissue behavior. In order to train our model to estimate breast volume deformation in response to pressure imposed with the US probe, we simulate several random probe-induced deformations using the following strategy:

- Select a random node $p$ in the breast surface

- Select an oriented bounding box $A$ centered in point $p$ and normal to the breast surface, whose dimensions match those of the US probe lower surface, which represents current probe-tissue contact area

- Select all the surface points $P$ falling within the box $A$

- Select as force direction $\boldsymbol{d}$ the normal to the surface at point $p$ plus a random angle $\alpha\left(\alpha \in\left[-\frac{\pi}{4}, \frac{\pi}{4}\right]\right)$

- Apply the same force $\boldsymbol{f}$ of random magnitude $(|f| \in[0.0,0.8])$ along direction $\boldsymbol{d}$ to the $P$ selected points simultaneously

- Store the displacement at the set of points $P$ (input to the network) and the displacement of all the points in the volume (output to the network) 
- Repeat the procedure until $N+M$ samples are generated

The choice of applying force $\boldsymbol{f}$ allowing some angle deviation from normal direction enables us to include in our dataset samples where the probe compression is not precisely normal to the surface, as it can be the case in freehand US acquisitions. The maximal force magnitude (e.g. $0.8 N$ ) is set such that the amount of maximal deformation reproduced in the training dataset never exceeds too much that observed in real clinical settings. The described strategy is used to generate the set $\left\{\left(\mathbf{u}_{\mathbf{s}}{ }^{n}, \mathbf{u}_{\mathbf{v}}{ }^{n}\right)\right\}_{n=1}^{N}$ of $N$ samples which is used to train the network, and the set $\left\{\left(\mathbf{u}_{\mathbf{s}}{ }^{n}, \mathbf{u}_{\mathbf{v}}{ }^{n}\right)\right\}_{n=1}^{M}$ of $M$ samples which is left for validation. The training dataset is generated with the SOFA framework [3] on a laptop equipped with an Intel i7-8750H processor and 16GB RAM.

\section{Experiments and Results}

The network presented in this work is used to predict US probe-induced deformations of a realistic multi-modality breast phantom (Model 073; CIRS, Norfolk, VA, USA). The 3D geometry model of the phantom surface and 10 inner lesions (diameter of $5-10 \mathrm{~mm}$ ) is obtained by segmenting the corresponding CT image, relying on ITK-SNAP and MeshLab frameworks [24, 2]. A Freehand Ultrasound System (FUS) based on a Telemed MicrUs US device (Telemed, Vilnius, Lithuania) equipped with a linear probe (model L12-5N40) is used to acquire US images of the 10 segmented lesions. The dimension of the probe surface is $(5 \times 1 \mathrm{~cm})$. For each lesion, we acquire US images in correspondence of four different input deformations. The MicronTracker Hx40 (ClaronNav, Toronto, Canada) optical tracking system is used to track US probe in space (Fig. 3(a)). The overall probe spatial calibration error is below $1 \mathrm{~mm}( \pm 0.7147)$, estimated through the PLUS toolkit [12]. Landmark-based rigid registration is performed to refer the CT-extracted 3D model, the US probe and the US images to the same common coordinate system, exploiting 3D Slicer functionalities [4]. The registration process does not only enable us to extract the breast-probe contact area, as described in Section 2, but also to know in real-time the 3D position of any point belonging to the US image. In this way, it is possible to refer lesions position extracted from US images to the 3D space.

\subsection{Predict displacement on synthetic data sets}

Elastic properties of the physics model used to generate training data are set in accordance with the values estimated in [23] for the same breast phantom considered in this study. However, as we are imposing surface displacements, the values of the elasticity parameters do not affect the displacement field inside the simulated volume as long as the ratio of the different stiffness values is maintained [16], thus making the method reliable for any patient specificity. Dirichlet boundary conditions are imposed by constraining the motion of all the nodes belonging to the lowest phantom surface. 


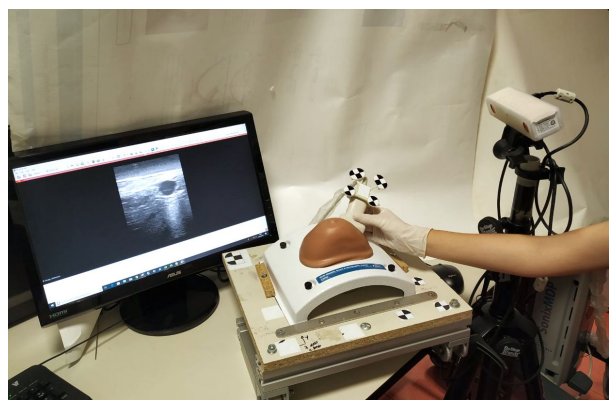

(a)

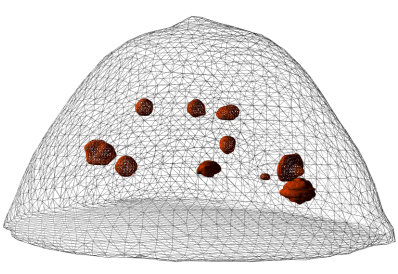

(b)

Fig. 3. (a)Experimental setup. From left to right: monitor showing real-time US images; CIRS breast phantom during FUS acquisition; optical tracking system that allows to map the real positions of the CIRS breast phantom and the US probe to the preoperative geometry model. (b)External surface and inner lesions of the CIRS breast phantom.

Using the method described in Sect. 2.2 and 2.3, we discretized the breast phantom into 2174 hexahedral elements and we simulated several probe-induced displacements. Overall we generated $N=800$ samples for training and $M=200$ samples for testing. The U-Net is trained in a GeForce GTX $1080 \mathrm{Ti}$ using a batch size of 4, 100000 iterations and the Adam optimizer. We used a Pytorch implementation of the U-Net. To assess the learning capability of the network, we perform a statistical analysis of the mean norm error e over the testing data set. Let $\mathbf{u}_{\mathbf{v}}{ }^{m}$ be the ground truth displacement tensor for sample $m$ generated using the finite element method described in Sect. 2.2 and $f\left(\mathbf{u}_{\mathbf{s}}{ }^{m}\right)$ the U-Net prediction. The mean norm error between $\mathbf{u}_{\mathbf{v}}{ }^{m}$ and $f\left(\mathbf{u}_{\mathbf{s}}{ }^{m}\right)$ for sample $m$ reads as:

$$
e\left(\mathbf{u}_{\mathbf{v}}{ }^{m}, f\left(\mathbf{u}_{\mathbf{s}}{ }^{m}\right)\right)=\frac{1}{n} \sum_{i=1}^{n}\left|\mathbf{u}_{\mathbf{v} i}{ }^{m}-f\left(\mathbf{u}_{\mathbf{s}}{ }^{m}\right)_{i}\right| .
$$

where $n$ is the number of degrees of freedom of the mesh. We compute the average $\bar{e}$, standard deviation $\sigma(e)$ and maximal value of such norm over the testing data set. The obtained results are shown in Table 1 . The maximal error is of only $0.266 \mathrm{~mm}$ and corresponds to the sample shown in Fig. 4(b). The most striking result is the small computation time required to make the predictions: only $3.14 \pm 0.56 \mathrm{~ms}$. In contrast, the FE method takes on average $407.7 \pm 64 \mathrm{~ms}$ to produce the solution. Obviously, the resolution of the FE mesh could be reduced to accelerate the computations but at the cost of an accuracy loss.

\subsection{Predict displacement on phantom data}

In our experiments, we consider one lesion at a time and we reposition the US probe on the surface of the breast such that the lesion considered is visible on the US image. In order to validate our model, we manually extract lesions position 


\begin{tabular}{|c|c|c||c|c|}
\hline $\begin{array}{c}\bar{e} \\
(\mathrm{~mm})\end{array}$ & $\begin{array}{c}\sigma(e) \\
(\mathrm{mm})\end{array}$ & $\begin{array}{c}\max _{m \in M} e \\
(\mathrm{~mm})\end{array}$ & $\begin{array}{c}\text { Prediction } \\
\text { time }(\mathrm{ms})\end{array}$ & $\begin{array}{c}\text { Total training } \\
\text { time }(\mathrm{min})\end{array}$ \\
\hline 0.052 & 0.050 & 0.266 & $3.14 \pm 0.56$ & 278 \\
\hline
\end{tabular}

Table 1. Error measures over the testing data set for a breast having 2174 H8 elements, with maximal nodal deformation of $79.09 \mathrm{~mm}$.

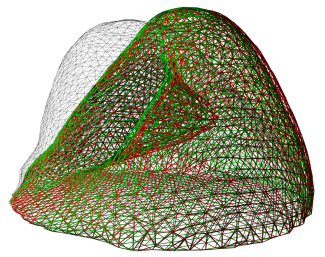

(a)

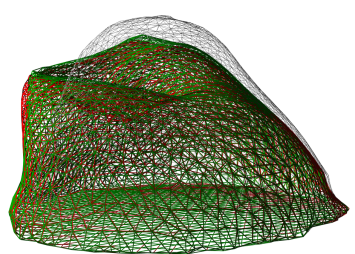

(b)

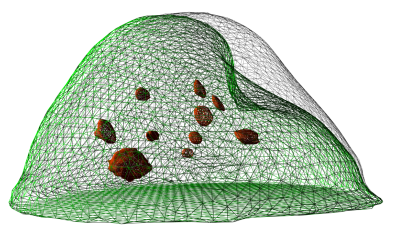

(c)

Fig. 4. (a) Sample with maximal deformation $(79.09 \mathrm{~mm})$. (b) Sample with maximal mean norm error $(0.266 \mathrm{~mm})$. The green mesh is the U-Net prediction and the red mesh is the FEM solution. The initial rest shape is shown in grey. (c) U-Net prediction on phantom data.

from US image acquired at rest (i.e., without applying any deformation, when the probe is only slightly touching the surface) and we consider it as a landmark to track. We then impose four deformations of increasing extent for each lesion, and we compare the U-Net-predicted displacement with real displacements extracted from US images. The comparison is performed computing target registration error (TRE) between the predicted position of the lesion and its ground-truth position. The performance of our method is compared to that of the FE model used for data generation. In Table 2 are shown the target registration errors for each phantom lesion with respect to the applied deformation. The input deformations are classified into five ranges based on the probe displacements. Displacement ranges indicated as D15, D20 and D25 have a fixed length of 5 $\mathrm{mm}$ each and are centered at 15, 20 and $25 \mathrm{~mm}$ respectively. D10 and D30 contain the extreme cases under $12.5 \mathrm{~mm}$ or above $27.5 \mathrm{~mm}$.

Values in Table 2 highlight that the average TRE for all the tumors and for all the deformations is smaller than $6.194 \mathrm{~mm}$ which is comparable to the maximum value obtained with the FE method $(6.080 \mathrm{~mm})$. The average error increases with the deformation range just like in the FE method. There is no significant difference between the values of the two tables, meaning that in terms of accuracy, our method is comparable to the data generation method used to train it. In order to compute each deformation, the FE method needs about $407.7 \mathrm{~ms}$ whereas the U-Net predicts the deformation in only $3 \mathrm{~ms}$. 
Table 2. Target registration errors in millimeters for different tumors and different deformation ranges in the breast phantom. The first table is for the proposed method, while the second table reports results obtained with the FE model used for data generation. Not-acquired data is reported as (-).

\begin{tabular}{|c|c|c|c|c|c|c|c|}
\hline \multicolumn{8}{|c|}{ U-Net predictions } \\
\hline TumorID & D10 & D15 & D20 & D25 & D30 & Mean & STD \\
\hline 1 & - & 1.936 & 2.002 & 1.506 & 3.053 & 2.124 & 0.569 \\
\hline 2 & 3.211 & 2.905 & 4.068 & - & 4.137 & 3.580 & 0.534 \\
\hline 3 & 2.032 & - & 4.709 & 7.134 & 10.90 & 6.194 & 3.262 \\
\hline 4 & 0.505 & 2.225 & 5.313 & 5.903 & - & 3.486 & 2.217 \\
\hline 5 & 0.932 & 2.768 & 3.454 & - & 4.893 & 3.012 & 1.425 \\
\hline 6 & 3.923 & 6.349 & 5.625 & - & 6.724 & 5.655 & 1.075 \\
\hline 7 & 3.454 & 3.864 & 4.543 & 6.710 & - & 4.643 & 1.255 \\
\hline 8 & 2.422 & 3.261 & 4.320 & 5.136 & - & 3.785 & 1.030 \\
\hline 9 & - & 3.928 & 4.214 & 4.578 & 4.858 & 4.394 & 0.353 \\
\hline 10 & 5.529 & 3.272 & 3.940 & 4.846 & - & 4.397 & 0.860 \\
\hline Mean & 2.751 & 3.390 & 4.219 & 5.116 & 5.761 & & \\
\hline STD & 1.638 & 1.294 & 1.007 & 1.854 & 2.788 & & \\
\hline \multicolumn{8}{|c|}{ FE method } \\
\hline TumorID & D10 & D15 & D20 & D25 & D30 & Mean & STD \\
\hline 1 & - & 1.326 & 2.151 & 2.075 & 3.759 & 2.328 & 0.887 \\
\hline 2 & 1.956 & 2.738 & 3.945 & - & 4.025 & 3.166 & 0.865 \\
\hline 3 & 1.595 & - & 4.748 & 7.044 & 10.932 & 6.080 & 3.404 \\
\hline 4 & 0.755 & 1.991 & 4.544 & 5.120 & - & 3.103 & 1.795 \\
\hline 5 & 1.029 & 2.863 & 3.330 & - & 4.541 & 2.941 & 1.262 \\
\hline 6 & 2.579 & 3.409 & 2.871 & - & 2.337 & 2.799 & 0.400 \\
\hline 7 & 2.605 & 3.219 & 4.095 & 6.750 & - & 4.167 & 1.582 \\
\hline 8 & 2.695 & 2.748 & 4.321 & 5.411 & - & 3.794 & 1.139 \\
\hline 9 & - & 2.745 & 2.497 & 2.510 & 4.193 & 2.986 & 0.704 \\
\hline 10 & 2.916 & 2.542 & 3.015 & 3.868 & - & 3.085 & 0.485 \\
\hline Mea & 2.016 & 2.620 & 3.552 & 4.682 & 4.964 & & \\
\hline STD & 0.765 & 0.593 & 0.856 & 1.803 & 2.757 & & \\
\hline
\end{tabular}

\section{Conclusion}

In this work we have proposed to use a deep neural network to learn the deformable behavior of the breast from numerical simulations based on the finite element method, in order to bypass the high computational cost of the FEM. Our approach represents an interface between precise biomechanical FE modeling (not capable of real time) and clinical applications requiring both high accuracy and very high speed. We have shown that our framework allows for extremely fast predictions of US probe-induced displacements of the breast during US scanning, achieving comparable accuracy to other existing methods. Therefore, it has the potential to be employed to update in real-time the estimated position of breast lesions identified on a pre-operative scan on US images, en- 
abling continuous visualization of the biopsy target, even when sonography fails to render it.

Although the FE model used to train our network does not perform in realtime, its prediction delay of less than $1 s$ might be considered already acceptable for our specific application. However, such good computational performance is achieved since in this preliminary evaluation we use a very simplistic model, that does not account for heterogeneity or complex boundary conditions happening in clinical cases. Usage of a more complex FE model will certainly cause an increase of computation load. On the contrary, an important feature of our approach is that the prediction time remains close to $3 \mathrm{~ms}$ regardless of the grid resolution and of the biomechanical model used for the data generation process. This means that increasing the complexity of the model used to generate the data set will not affect the prediction speed. Moreover, our pipeline allows the method to be insensitive to patient specific elastic properties as it imposes surface displacements. It is worth noting that for inhomogeneous objects, the displacement field still depends on the ratio of the different stiffnesses [16]. Another advantage of our method is the easy meshing process. Any geometry can be embedded in a sparse grid and through the use of immersed boundary simulations the deformations are correctly estimated.

The main limitation of our method remains the training process, which is burdersome and has to be repeated for every new geometry or application. However, we have shown that a limited amount of training data can be sufficient to train a U-Net such that it obtains accurate prediction within clinically acceptable times. As a future work, we plan to use a more general training strategy leading to a network model able to predict deformations induced by any type and number of compression tools (for example, different probe shapes or the two biopsy compression plates).

\section{Bibliography}

[1] Brunet, J.N., Mendizabal, A., Petit, A., Golse, N., Vibert, E., Cotin, S.: Physics-based deep neural network for augmented reality during liver surgery. MICCAI (2019)

[2] Cignoni, P., Callieri, M., Corsini, M., Dellepiane, M., Ganovelli, F., Ranzuglia, G.: MeshLab: an Open-Source Mesh Processing Tool. In: Scarano, V., Chiara, R.D., Erra, U. (eds.) Eurographics Italian Chapter Conference. The Eurographics Association (2008)

[3] Faure, F., Duriez, C., Delingette, H., et al.: Sofa: A multi-model framework for interactive physical simulation. In: Soft tissue biomechanical modeling for computer assisted surgery, pp. 283-321. Springer (2012)

[4] Fedorov, A., Beichel, R., Kalpathy-Cramer, J., Finet, J., Fillion-Robin, J.C., Pujol, S., Bauer, C., Jennings, D., Fennessy, F., Sonka, M., Buatti, J., Aylward, S., Miller, J., Pieper, S., Kikinis, R.: 3d slicer as an image computing 
platform for the quantitative imaging network. Magnetic resonance imaging 30(9), 1323-1341 (2012)

[5] Felippa, C.A., Haugen, B.: A unified formulation of small-strain corotational finite elements: I. theory. Computer Methods in Applied Mechanics and Engineering 194(21-24), 2285-2335 (2005)

[6] Guo, R., Lu, G., Qin, B., Fei, B.: Ultrasound imaging technologies for breast cancer detection and management: A review. Ultrasound in medicine \& biology (2017)

[7] Han, L., Hipwell, J.H., Eiben, B., et al.: A nonlinear biomechanical model based registration method for aligning prone and supine $\mathrm{mr}$ breast images. IEEE transactions on medical imaging 33(3), 682-694 (2013)

[8] Hipwell, J.H., Vavourakis, V., Han, L., et al.: A review of biomechanically informed breast image registration. Physics in Medicine \& Biology 61(2), R1 (2016), http://stacks.iop.org/0031-9155/61/i=2/a=R1

[9] Hu, Y., Ahmed, H.U., Taylor, Z., Allen, C., Emberton, M., Hawkes, D., Barratt, D.: Mr to ultrasound registration for image-guided prostate interventions. Medical image analysis 16(3), 687-703 (2012)

[10] Joldes, G.R., Wittek, A., Miller, K.: Real-time nonlinear finite element computations on gpu-application to neurosurgical simulation. Computer methods in applied mechanics and engineering 199(49-52), 3305-3314 (2010)

[11] Krücker, J., Xu, S., Venkatesan, A., Locklin, J.K., Amalou, H., Glossop, N., Wood, B.J.: Clinical utility of real-time fusion guidance for biopsy and ablation. J Vasc Interv Radiol 22(4), 515-524 (2011)

[12] Lasso, A., Heffter, T., Rankin, A., Pinter, C., Ungi, T., Fichtinger, G.: Plus: open-source toolkit for ultrasound-guided intervention systems. IEEE Transactions on Biomedical Engineering 61(10), 2527-2537 (2014)

[13] Marchesseau, S., Heimann, T., Chatelin, S., Willinger, R., Delingette, H.: Multiplicative jacobian energy decomposition method for fast porous viscohyperelastic soft tissue model. In: MICCAI. pp. 235-242. Springer (2010)

[14] Martínez-Martínez, F., Rupérez-Moreno, M.J., Martínez-Sober, M., et al.: A finite element-based machine learning approach for modeling the mechanical behavior of the breast tissues under compression in real-time. Computers in biology and medicine 90, 116-124 (2017)

[15] Miller, K., Joldes, G., Lance, D., Wittek, A.: Total lagrangian explicit dynamics finite element algorithm for computing soft tissue deformation. Communications in numerical methods in engineering 23(2), 121-134 (2007)

[16] Miller, K., Lu, J.: On the prospect of patient-specific biomechanics without patient-specific properties of tissues. Journal of the mechanical behavior of biomedical materials 27, 154-166 (2013)

[17] Morooka, K., Chen, X., Kurazume, R., Uchida, S., Hara, K., Iwashita, Y., Hashizume, M.: Real-time nonlinear fem with neural network for simulating soft organ model deformation. In: MICCAI. pp. 742-749. Springer (2008)

[18] O'Flynn, E., Wilson, A., Michell, M.: Image-guided breast biopsy: state-ofthe-art. Clinical radiology 65(4), 259-270 (2010)

[19] Pfeiffer, M., Riediger, C., Weitz, J., Speidel, S.: Learning soft tissue behavior of organs for surgical navigation with convolutional neural networks. IJCARS pp. 1-9 (2019) 
[20] Ronneberger, O., Fischer, P., Brox, T.: U-net: Convolutional networks for biomedical image segmentation. MICCAI pp. 234-241 (2015)

[21] Tagliabue, E., Dall'Alba, D., Magnabosco, E., Tenga, C., Peterlik, I., Fiorini, P.: Position-based modeling of lesion displacement in ultrasound-guided breast biopsy. IJCARS (2019)

[22] Tonutti, M., Gras, G., Yang, G.Z.: A machine learning approach for realtime modelling of tissue deformation in image-guided neurosurgery. Artificial intelligence in medicine 80, 39-47 (2017)

[23] Visentin, F., Groenhuis, V., Maris, B., et al.: Iterative simulations to estimate the elastic properties from a series of mri images followed by mri-us validation. Medical and biological engineering and computing 194(21-24), 1-12 (2018)

[24] Yushkevich, P.A., Piven, J., Cody Hazlett, H., et al.: User-guided 3D active contour segmentation of anatomical structures: Significantly improved efficiency and reliability. Neuroimage 31(3), 1116-1128 (2006) 